Johannes Amann*, Tobias Baur, Caroline Schultealbert und Andreas Schütze

\title{
Bewertung der Innenraumluftqualität über VOC-Messungen mit Halbleitergassensoren - Kalibrierung, Feldtest, Validierung
}

\author{
Evaluation of indoor air quality via VOC measurements with MOS gas sensors - calibration, field \\ test, validation
}

DOI 10.1515/teme-2021-0058

Zusammenfassung: In jüngster Vergangenheit sind immer mehr Halbleitergassensoren im Bereich Innenraumluftqualität auf den Markt gekommen. Diese messen die Gesamtbelastung an flüchtigen organischen Komponenten (engl.: volatile organic compounds, kurz: VOCs) oder einen relativen Innenraumluftqualitätsindex. Mit einer optimierten Betriebsweise und Kalibrierung können die Sensoren in ihrer Selektivität und Sensitivität gesteigert werden, was für die Bewertung der Innenraumluftqualität wichtig wäre. Die Möglichkeiten und Grenzen werden in dieser Studie vorgestellt. Für diese Zielanwendung müssen die Sensoren zuerst kalibriert werden. Auf Basis der Daten einer umfangreichen Laborkalibrierung kommen verschiedene Methoden des maschinellen Lernens für die Datenauswertung zum Einsatz. Diese Studie beinhaltet die Untersuchung der Eignung eines kommerziell verfügbaren Multisensors (SGP30, Sensirion) im temperaturzyklischen Betrieb für die selektive Messung von VOCs im Innenraum. Nach der Laborkalibrierung wurde der Sensor vier Wochen im Feld betrieben. Über Freisetzungstests durch Verdampfung von Flüssigkeiten wurden die Modelle auf Funktionalität geprüft. Außerdem wurden mit den analytischen Messsystemen TD-GC-MS (ThermodesorptionGaschromatographie-Massenspektrometer mit Tenax Probennahme) und einem mobilen GC mit Photo-IonisationsDetektor, PID (X-pid 9500, Dräger) regelmäßig Vergleichsmessungen durchgeführt. Mithilfe von Rekalibrierungen im Labor und anschließenden Feldtests wurde außerdem die Stabilität der Modelle untersucht. Diese Studie zeigt, dass mit dem SGP30 eine Vor-Ort-Messung der Gesamt-VOCBelastung in Innenräumen mit hoher zeitlicher Auflösung

\footnotetext{
*Korrespondenzautor: Johannes Amann, Lehrstuhl für Messtechnik, Universität des Saarlandes, Campus A5 1, 66123 Saarbrücken, E-Mail: j.amann@lmt.uni-saarland.de Tobias Baur, Caroline Schultealbert, Andreas Schütze, E-Mail: t.baur@lmt.uni-saarland.de, c.schultealbert@lmt.uni-saarland.de schuetze@lmt.uni-saarland.de
}

möglich ist und auch eine selektive Messung bestimmter Stoffe und Stoffgruppen realisierbar ist.

Schlüsselwörter: Halbleitergassensor, VOC flüchtige organische Verbindungen, IAQ Innenraumluftqualität

Abstract: In the recent past more and more metal oxide semiconductor (MOS) gas sensors for monitoring indoor air quality (IAQ) have been introduced in the market. The aim is to measure indoor the total amount of volatile organic compounds (VOCs) or a relative IAQ index. With an optimized operating mode and calibration, the sensors selectivity and sensitivity can be increased, which is highly important for IAQ evaluation. Their potential and limits are presented in this study. For this application, the sensors first have to be calibrated. In addition to extensive lab calibration, various machine learning methods are used for data evaluation. This study presents an investigation of the suitability of a commercial multisensor (SGP30, Sensirion) in temperature-cycled operation. After lab calibration the sensor was operated in the field over four weeks. The models were checked for functionality via release tests of target substances. In addition, analytical measurements were carried out as often as possible with TD-GC-MS (thermal desorption - gas chromatography mass spectrometry using Tenax sampling) and a mobile GC-PID (X-pid 9500, Dräger). The stability was also investigated by performing recalibrations in the lab and subsequent field tests. This study shows that the SGP30 allows on-site monitoring of the total VOC pollution indoors with high temporal resolution and also selective measurements of certain individual substance groups.

Keywords: MOS metal oxide semiconductor gas sensor, VOC volatile organic compunds, IAQ indoor air quality 


\section{Einleitung}

Nach einem WHO-Bericht aus dem Jahr 2013 verbringen Menschen $90 \%$ ihrer Zeit in Innenräumen [15]. Dabei wird unterschätzt, dass die Innenraumluft häufig stärker belastet ist als die Außenluft $[12,13]$. Viele Schadstoffe in Innenräumen sind VOCs, welche von Baumaterialien und Einrichtungsgegenständen, durch Vorgänge wie Reinigen, Kochen, Heizen oder durch den menschlichen Stoffwechsel freigesetzt werden $[4,5,12]$. Die verbreitete $\mathrm{CO}_{2}$-Sensorik ist lediglich als Indikator für die vom Menschen emittierten VOCs geeignet und bietet keine umfassende Bewertung der Schadstoffbelastung in Innenräumen. In der Analytik werden Innenräume nach ISO 16000-6 dagegen ohne Anwesenheit des Menschen vermessen [6]. Im Gegensatz dazu können mit Halbleitergassensoren, die eine direkte Messung von VOCs ermöglichen, alle Quellen berücksichtigt und Vor-Ort-Messungen mit hoher Zeitauflösung realisiert werden.

Wir präsentieren eine der ersten Studien zur Bewertung der Innenraumluftqualität mit Halbleitergassensoren durch Erfassung der Gesamt-VOC-Belastung und gleichzeitiger Quantifizierung einzelner VOC-Stoffgruppen und Substanzen außerhalb des Labors. Diese umfasst die Kalibrierung der Sensoren im Labor, anschließende Feldtests und gezielte Freisetzungen von VOCs, um die durch die Kalibrierung erzielte Selektivität und die korrekte Quantifizierung nachzuweisen. Zudem wird die Stabilität der Sensoren betrachtet. Zusätzlich werden zum Vergleich umfangreiche analytische Messungen durchgeführt.

\section{Experimentelles}

\subsection{Apparativer Aufbau}

Hauptbestandteil dieser Studie sind Messungen mit dem Halbleitergassensor SGP30 der Firma Sensirion [14]. Dieser kombiniert vier sensitive Schichten mit integrierter Ausleseelektronik auf einem Sensorchip und ermöglicht durch Ansteuerung der Heizstruktur eine Temperatursteuerung, welche für den Betrieb des Sensors im temperaturzyklischen Betrieb benötigt wird [1]. Diese Betriebsweise ermöglicht eine deutliche Steigerung der Sensitivität und Selektivität sowie eine verbesserte Stabilität $[11,16]$. Der verwendete Temperaturzyklus mit einer Dauer von 120 Sekunden, bestehend aus zehn Hochtemperaturstufen gefolgt von jeweils zehn unterschiedlichen Niedrigtemperaturstufen, ist in Abb. 1 dargestellt.

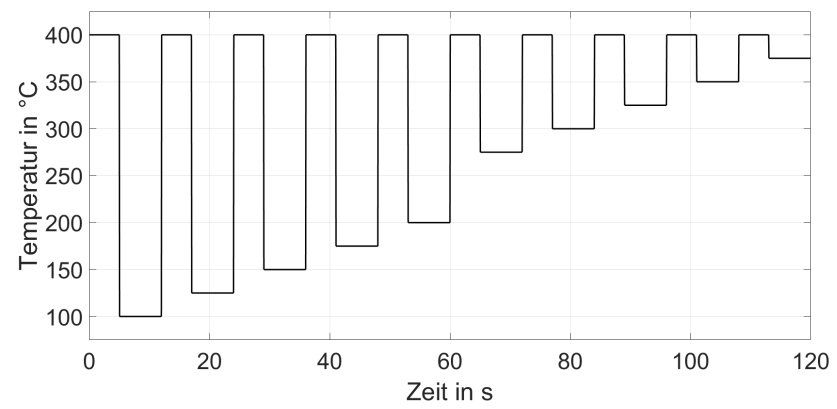

Abb. 1: Temperaturzyklus mit den Hochtemperaturstufen $\left(400^{\circ} \mathrm{C}\right)$ und Niedrigtemperaturstufen $(100,125,150,175,200,275,300$, $325,350,375^{\circ} \mathrm{C}$ )

\subsection{Kalibrierung und Rekalibrierung in der GMA}

Im ersten Schritt werden die Halbleitergassensoren im Labor für den Einsatz im Innenraum zur Quantifizierung von VOCs, speziell Gesamt-VOC sowie bestimmter Substanzgruppen, mit randomisierten Gasgemischen kalibriert [2]. Mit unserer Gasmischanlage (kurz: GMA) ist es möglich, sieben Zielgrößen, sechs Gase und Feuchte, gleichzeitig und unabhängig zu variieren [7]. Um die komplexe Zusammensetzung der Innenraumluft möglichst realitätsnah abzubilden, wurden als Zielgrößen vier VOCs (Aceton, Toluol, Ethanol, Formaldehyd) ausgewählt, welche in Innenräumen häufig die höchste Konzentration aufweisen. Gleichzeitig sollten damit vier Vertreter aus verschiedenen Stoffgruppen (Keton, Aromat, Alkohol, Aldehyd) kalibriert werden, da Gase einer Stoffgruppe auf Halbleitergassensoren durch die gleichen funktionellen Gruppen häufig ähnliche Reaktionen hervorrufen. Eine abgeleitete Zielgröße stellt die Summe der vier VOCs, kurz: VOC $_{\text {sum, }}$, dar, welche zur Abschätzung der Gesamtbelastung durch VOCs dient. Zusätzlich sind die Störgrößen Wasserstoff, Kohlenmonoxid und Feuchte Bestandteil der Kalibrierung, da diese im Innenraum variieren und Halbleitergassensoren sensitiv auf diese Gase reagieren.

Die Konzentrationsbereiche der Zielgrößen wurden anhand typischer Konzentrationen im Innenraum gewählt. Dafür wurden mehrere Studien mit analytischen Messungen von Innenräumen betrachtet, welche als Grundlage für den Standardkonzentrationsbereich nach Tab. 1 dienen [8, 9]. Die Zielgrößen, welche als Teil der Feldtests zur Validierung freigesetzt werden, werden zusätzlich in einem erweiterten Konzentrationsbereich bis zu 1 ppm (Aceton, Toluol, Ethanol) bzw. 4 ppm (Wasserstoff) angeboten. Insgesamt besteht eine Kalibrierung aus 500 Gasgemischen mit einer Dauer von jeweils 30 Minuten pro Gasgemisch, entsprechend einer Gesamtdauer von etwa einer Woche. 
Tab. 1: Standardkonzentrationsbereich

\begin{tabular}{cc}
\hline Zielgröße & Konzentrationsbereich \\
\hline Aceton & $14-300 \mathrm{ppb}$ \\
Toluol & $4-300 \mathrm{ppb}$ \\
Formaldehyd & $1-400 \mathrm{ppb}$ \\
Ethanol & $4-300 \mathrm{ppb}$ \\
VOC $_{\text {sum }}$ & $300-1200 \mathrm{ppb}$ \\
Wasserstoff & $400-2000 \mathrm{ppb}$ \\
Kohlenmonoxid & $150-2000 \mathrm{ppb}$ \\
Feuchte & $25-70 \%$ \\
\hline
\end{tabular}

Für die Untersuchung der Stabilität der Sensoren wurden zwischen und nach zwei Feldtestperioden Rekalibierungen mit dem beschriebenen Gasprofil durchgeführt.

\subsection{Datenauswertung}

Im Anschluss an die Kalibrierung wird für jede Zielgröße ein statistisches Modell zur Vorhersage der Konzentration im Feld gebildet. Aus dem Betrieb des SGP30 im Temperaturzyklus entsteht ein hochdimensionaler Datensatz.

Im ersten Schritt wird eine Merkmalsextraktion durchgeführt, indem formbeschreibende Merkmale (Mittelwert \& Steigung) aus dem Temperaturzyklus extrahiert werden. Bei Einteilung des Zyklus in 120 äquidistante Bereiche resultieren hieraus pro Sensorschicht je 120 Mittelwerte und Steigungsmerkmale, für einen SGP30 somit insgesamt 960 Merkmale.

Da hieraus hochkorrelierte Merkmale entstehen, ist eine Merkmalsselektion als zweiter Schritt vorgesehen. Deren Ziel ist, die Merkmale nach ihrer Bedeutung für die Quantifizierung der jeweiligen Zielgröße zu sortieren und dementsprechend die wichtigsten Merkmale auszuwählen.

Im abschließenden Schritt der Modellbildung erfolgt die eigentliche Quantifizierung durch die Regressionsmethode Partial Least Square Regression (kurz: PLSR).

Zur Validierung ist in der Modellbildung eine 10-fache Kreuzvalidierung integriert und $20 \%$ des Kalibrierungsdatensatzes werden für das Testen aus der Modellbildung zurückgehalten.

\subsection{Feldtests}

Für die Funktionsprüfung der Modelle außerhalb des Labors werden Feldtests in einem regulären Büro an der Universität des Saarlandes am Lehrstuhl für Messtechnik durchgeführt (Abb. 2).

Der Feldtestraum hat ein Volumen von $61,8 \mathrm{~m}^{3}$ und besitzt neben drei Arbeitsplätzen mit Schreibtischen und

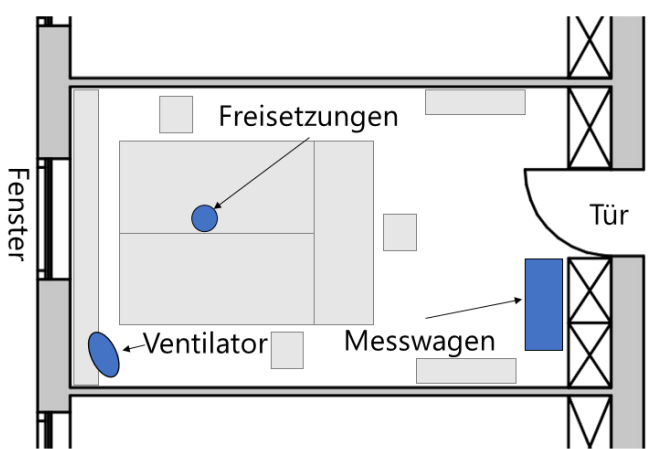

Abb. 2: Freisetzungsraum mit Markierung für den Ort der Freisetzungen, Positionierung des Ventilators und des Messwagens mit den Halbleitergassensoren und analytischen Messsystemen.

Bürostühlen einen Teppichboden und mehrere Schränke. Über eine Tür zum Gang und über ein Fenster kann der Raum gelüftet und mit frischer Außenluft versorgt werden. Durch Betrieb der Sensoren im geschlossenen Raum ohne Anwesenheit von Personen wird die Hintergrundbelastung aufgenommen.

Ergänzend dienen Freisetzungen der Zielsubstanzen durch Verdunstung von Flüssigkeiten zur weiteren Validierung der gebildeten Modelle. Der theoretische Konzentrationsanstieg bei einer Freisetzung kann bei homogener Verteilung des Gases im Raum ohne Luftaustausch und Interaktion mit Oberflächen über das ideale Gasgesetz berechnet werden. Begleitend wurden so oft wie möglich umfangreiche Messungen mit analytischen Messsystemen durchgeführt, u.a. mit einem GC-MS (Thermo Fisher Scientific) mit Tenax-Probennahme und einem mobilen GC-PID (Dräger X-pid 9500).

\section{Ergebnisse}

\subsection{Kalibrierung}

In Abb. 3 sind die Ergebnisse der Kalibrierung in Form der RMSE-Werte für Validierung und den Testdatensatz der Modellbildung für die sieben Zielgrößen dargestellt. Dabei werden Werte zwischen 10 ppb für Aceton und 80 ppb für Kohlenmonoxid erreicht. Eine Quantifizierung der Zielgrößen bis in den zweistelligen ppb-Bereich ist somit mit dieser Vorgehensweise zu erzielen. Lediglich Kohlenmonoxid sticht mit einem RMSE von über 50 ppb heraus. Vorhergende Untersuchungen haben gezeigt, dass keine der vier Schichten ein besondere Sensitivität auf Kohlenmonoxid aufweist.

Ein bekanntes Problem bei Halbleitergassensoren stellen Veränderungen der sensitiven Schicht und Instabilitä- 


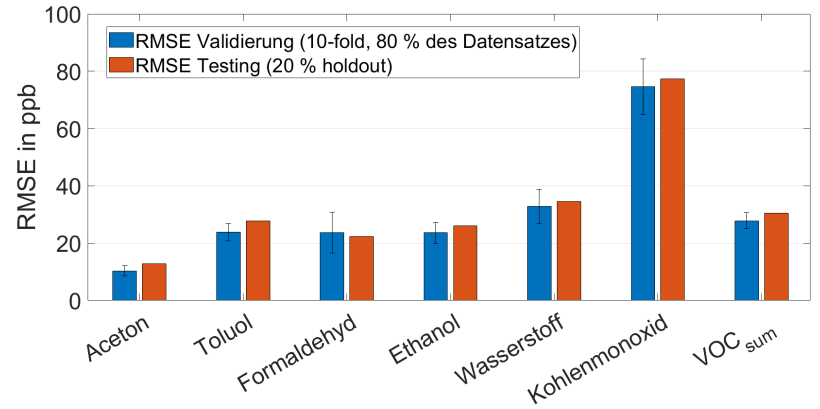

Abb. 3: Root Mean Square Error (RMSE) für Validierung und Test aus der Kalibrierung für die sieben Zielgrößen.

ten dar [10]. Daraus resultierte während der Feldtests ein Driften der Modellsignale. Zur Kompensation dieser Drifteffekte wurde ein Teil der 1. Rekalibrierung, welche nach den ersten vier Wochen des Feldtests durchgeführt wurde, zusätzlich als Trainingsdaten mit in die Modellbildung aufgenommen [3].

\subsection{Feldtests - kalibrierte VOCs}

Abbildung 4 zeigt zwei Freisetzungen von Stoffen, welche Teil der Kalibrierung und damit des Trainingsdatensatzes sind (Aceton und Toluol). Die Modellsignale der freigesetzten Substanzen erzeugen bei der Freisetzung einen deutlichen Hub (oben, im Vergleich zum mobilen GC-PID), während die restlichen Modellsignale mit Ausnahme von $\mathrm{VOC}_{\text {sum }}$ (unten) keinen Anstieg zeigen. Die analytischen Messungen bestätigen die durchgeführten Freisetzungen; dabei muss beachtet werden, dass die resultierenden Konzentrationen unterhalb der vom Hersteller angegebenen Bestimmungsgrenze des X-pid 9500 liegen. Die Freisetzungsmengen wurde jeweils so gewählt, dass im Raum ein theoretischer Konzentrationsanstieg von 600 ppb resultiert. Das Aceton-Modell des SGP30 zeigt eine Abweichung von $20 \%$, das Toluol-Modell von $5 \%$. Das VOC $_{\text {sum-Modell }}$ gleicht den beiden Einzelfreisetzungen sowohl vom Verlauf als auch von den absoluten Werten.

Abbildung 5 zeigt eine Ethanol-Freisetzung (links) und die Signale, die aus zwei aufeinander folgenden, gleichzeitigen Freisetzungen von Aceton und Toluol resultieren (rechts). Bei Ethanol zeigen wiederum das Ethanol-Modell

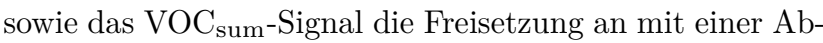
weichung von $10 \%$ im Vergleich zum theoretisch berechneten Konzentrationsanstieg. Die restlichen Modelle zeigen wiederum keine Reaktion auf die Ethanol-Freisetzung.

Die gleichzeitige Freisetzung von Aceton und Toluol wird mithilfe der beiden analytischen Messsysteme GC-MS und X-pid 9500 validiert. Alle Signale zeigen ein ähnliches
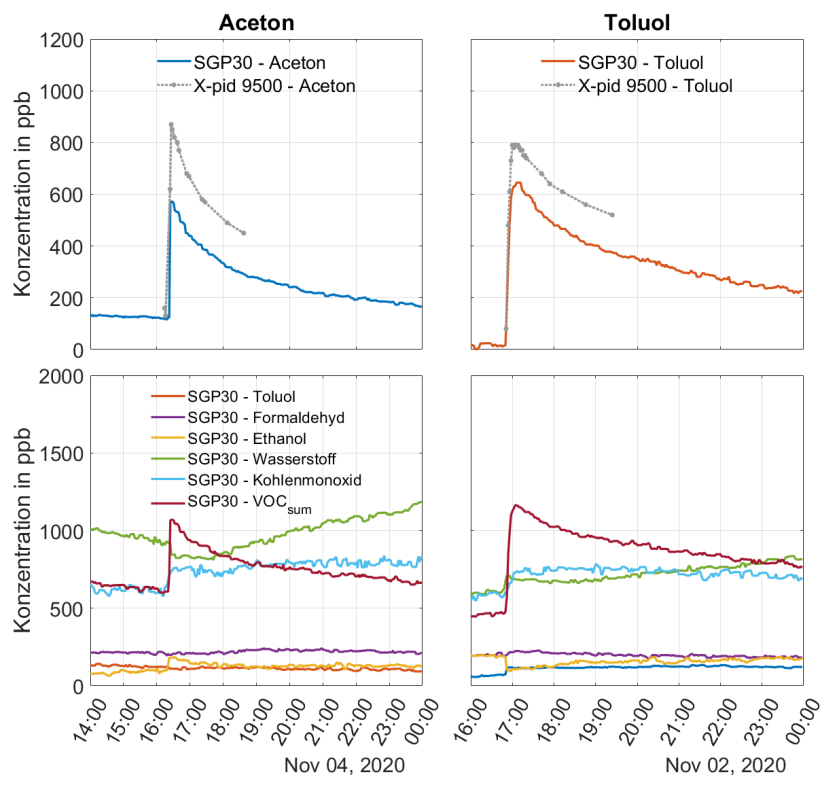

Abb. 4: Darstellung der Konzentrationsverläufe der modellbasierten Signale und des X-pid 9500 während der Freisetzung von Aceton (links) und Toluol (rechts). Der theoretische Konzentrationsanstieg resultierend aus der Freisetzungsmenge beträgt bei gleichmäßiger Verteilung im Raum jeweils ca. 600 ppb für beide Substanzen.

Verhalten und unterscheiden sich lediglich in der absoluten Höhe. Im Vergleich zum GC-MS überschätzt das SGP30Sensormodell die Konzentration um etwa 20\%. Das Xpid 9500 zeigt dagegen nochmals etwa $20 \%$ höhere Werte als das SGP30-Modell an. Welches Messsystem am nächsten an der wahren Konzentration im Innenraum liegt, ist nicht zu beurteilen, da die Konzentrationen der freigesetzten Substanzen unter der jeweiligen Bestimmungsgrenze des X-pid 9500 liegen und auch beim GC-MS Messunsicherheiten von bis zu $20 \%$ angegeben werden, zudem erfolgte dafür keine rückführbare Kalibrierung.

Besonders interessant ist die Betrachtung des

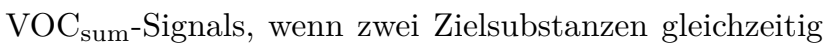
freigesetzt werden, aber unterschiedlich schnell verdunsten, wie es bei der zweiten Freisetzung von Aceton und Toluol der Fall ist. Wie gewünscht zeigt dieses Signal den gleichen Verlauf wie die Summe der Modelle für die EinzelVOCs. Hierbei dominiert zuerst der schnelle Anstieg der Aceton-Konzentration, bis das Aceton-Signal nach dem Maximum wieder sinkt, während das Toluol-Signal langsamer ansteigt und deshalb im weiteren Verlauf dominiert,

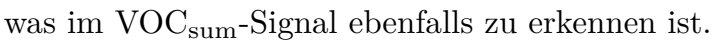

Das Wasserstoff-Signal zeigt nach Freisetzung von VOCs einen Anstieg, dessen Verhalten detailliert in einer weiteren Veröffentlichung dargestellt ist [17]. 


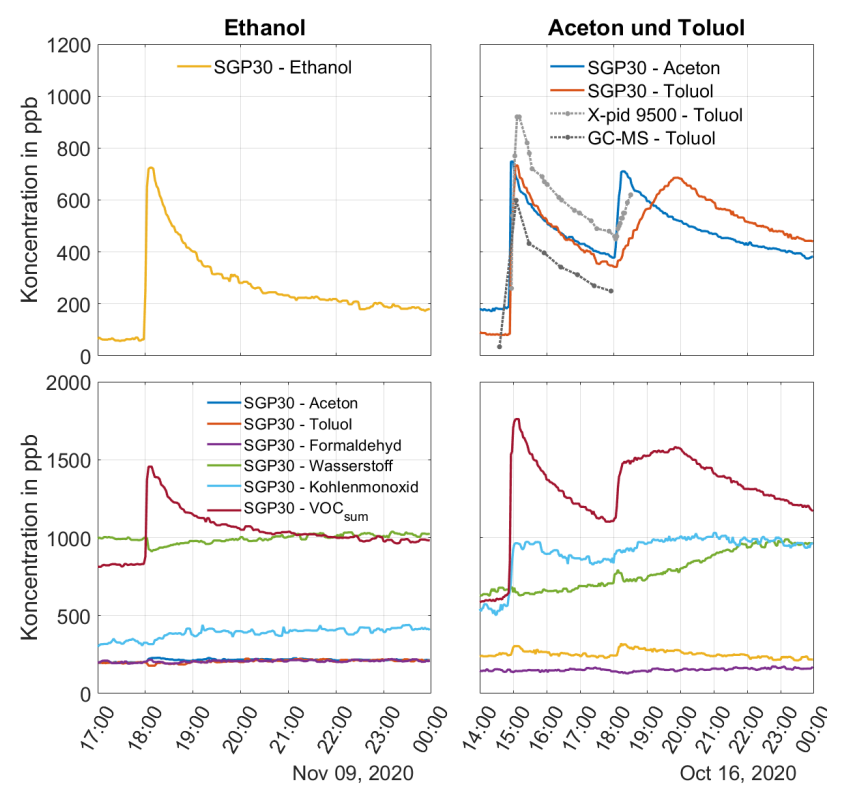

Abb. 5: Darstellung der Konzentrationsverläufe der modellbasierten Signale und der analytischen Messsystemen während der Freisetzung von Ethanol (links) und bei gleichzeitiger Freisetzung von Aceton und Toluol (rechts). Der theoretische Konzentrationsanstieg resultierend aus der Freisetzungsmenge beträgt bei gleichmäßiger Verteilung im Raum jeweils ca. 600 ppb für die Substanzen.

\subsection{Feldtests - unkalibrierte VOCs}

Ein weiterer untersuchter Aspekt ist das Verhalten der VOC-Modelle bei Freisetzungen von Stoffen, welche nicht Teil der Kalibrierung waren. In Abb. 6 sind die Modellsignale während einer Freisetzung von Xylol, das wie Toluol zu den Aromaten zählt, dargestellt. Aus der Xylol-Freisetzung resultiert ein Anstieg des Toluol-Signals (links), was bestätigt, dass beide Aromate ähnliche Reaktionen bzw. Responsemuster auf dem Sensor auslösen. Eine Abweichung ist aber bei der absoluten Höhe des Signals zu erkennen: bei der Freisetzung von 600 ppb Xylol zeigt das Toluol-Modell einen Anstieg von rund $450 \mathrm{ppb}$ an, während bei der Toluol-Freisetzung ein Anstieg von rund $650 \mathrm{ppb}$ resultiert. Auch bei der gleichzeitigen Freisetzung von $600 \mathrm{ppb}$ Toluol und $600 \mathrm{ppb}$ Xylol (rechts) reagiert das Toluol-Modell auf beide Freisetzungen mit einem Anstieg von knapp 1000 ppb. In beiden Fällen zeigt auch das $\mathrm{VOC}_{\text {sum-Modell die gleichen Verläufe wie das }}$ Toluol-Modell mit ähnlichen Werten. Zumindest für diese beiden Vertreter der Aromaten kann somit festgehalten werden, dass das Toluol-Modell als Stoffgruppen-Signal dient.

Für Alkohole ergibt sich bei der Freisetzung von Ethanol und Isopropanol ein anderes Bild, wie in Abb. 7 dar-
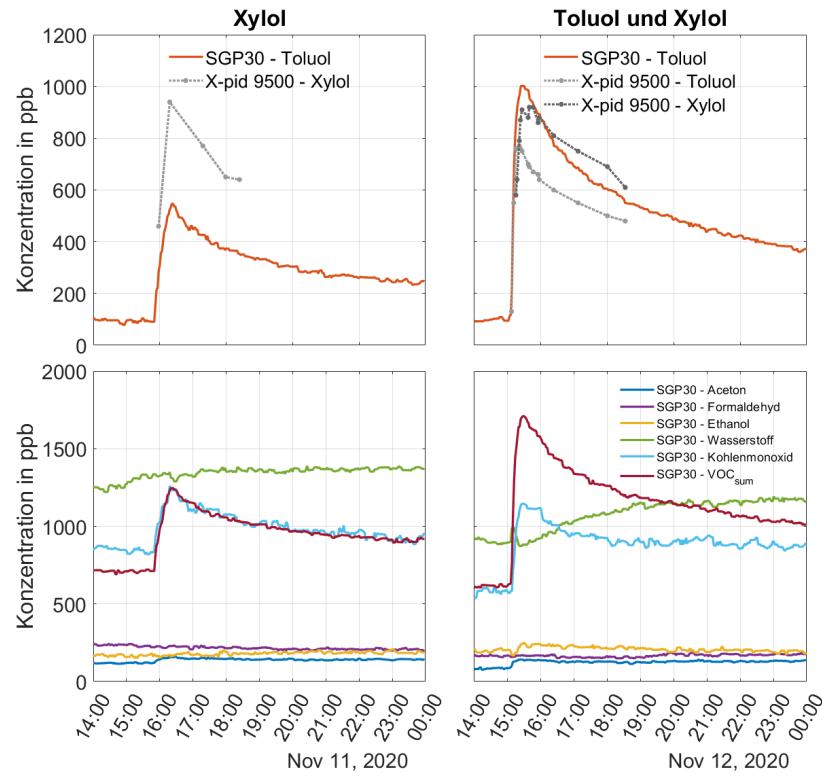

Abb. 6: Darstellung der Konzentrationsverläufe der modellbasierten Signale und des X-pid 9500 während der Freisetzung von Xylol (links) und Toluol und Xylol (rechts). Der theoretische Konzentrationsanstieg resultierend aus der Freisetzungsmenge beträgt bei gleichmäßiger Verteilung im Raum jeweils ca. 600 ppb für beide Substanzen.

gestellt. Das Ethanol-Signal wie auch das $\mathrm{VOC}_{\text {sum-Signal }}$ reagieren nicht auf die Freisetzung von Isopropanol. Die Freisetzung wurde mit dem X-pid 9500 verifiziert und auch die Rohsignale des SGP30 zeigen eine Reaktion auf Isopropanol, jedoch unterscheiden sich die Muster im Sensorsignal im Vergleich zur Freisetzung von Ethanol deutlich. Dieses Ergebnis zeigt aber, dass eine selektive Quantifizierung von Ethanol auch bei Anwesenheit von Isopropanol möglich ist. Ob dies auch für weitere Vertreter der Alkohole zutrifft, muss weiter untersucht werden, ebenso wie sich die Sensormodelle für andere VOC-Klassen verhalten.

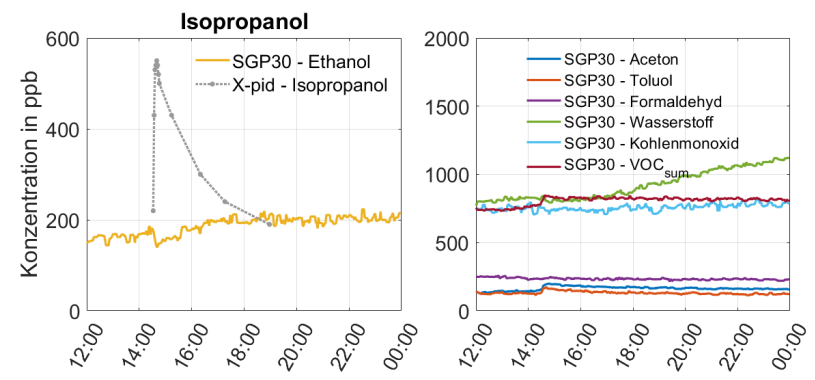

Abb. 7: Darstellung der Konzentrationsverläufe der modellbasierten Signale und des X-pid 9500 während der Freisetzung von Isopropanol. Der theoretische Konzentrationsanstieg resultierend aus der Freisetzungsmenge beträgt bei gleichmäßiger Verteilung im Raum ca. 600 ppb. 


\section{Zusammenfassung}

Die vorliegende Studie zeigt das große Potential von Halbleitergassensoren im temperarturzyklischen Betrieb zur Erfassung der Innenraumluftqualität. Mit der vorgestellten Kalibrierungsmethode, der Verwendung des temperaturzyklischen Betriebs und der Datenauswertung ist es möglich, statistische Modelle zu bilden, mit denen eine Quantifizierung von VOCs im Innenraum ab Konzentrationen im zweistelligen ppb-Bereich ermöglicht wird. Die Modelle wurden validiert und ihre Stabilität untersucht im Rahmen von Feldtests mit gezielten Freisetzungen begleitet durch Vergleichsmessungen mit analytischen Messsystemen. Im Vergleich zu den analytischen Messsystemen wird mit den deutlich kostengünstigeren sensorbasierten Systemen eine ähnliche Messqualität erzielt, zudem sind diese echtzeitfähig, z. B. für eine bedarfsgerechte Lüftung. Neben der Erfassung der Gesamt-VOC-Belastung in Innenräumen besteht zudem das Potential zur Quantifizierung einzelner, besonders gesundheitsschädlicher Substanzen.

Zukünftig sollen mehr Stoffgruppen und auch mehr Vertreter pro Stoffgruppe in die Kalibrierung einbezogen werden, um die komplexe Umgebung im Innenraum besser nachbilden zu können. Zusätzlich sollen in weiteren Feldtests realitätsnahe Szenarien untersucht werden, z. B. Anwesenheit von Menschen oder Tätigkeiten wie Reinigen, Kochen oder Renovierungen.

Danksagung: Ein Teil dieser Forschung wurde im Rahmen des vom Europäischen Fonds für regionale Entwicklung (EFRE) finanzierten Projekts „SE-ProEng“ durchgeführt. Wir danken der Deutschen Forschungsgemeinschaft (DFG) und der Universität des Saarlandes für die Unterstützung im Rahmen des Förderprogramms Open Access Publishing. Wir danken der Dräger Safety AG \& Co KGaA für die Bereitstellung des X-pid 9500 für diese Studie.

\section{Literatur}

[1] T. Baur, A. Schütze und T. Sauerwald. Optimierung des temperaturzyklischen Betriebs von Halbleitergassensoren. tm-Technisches Messen, 82(4):187-195, 2015.

[2] T. Baur, M. Bastuck, C. Schultealbert, T. Sauerwald und A. Schütze. Random gas mixtures for efficient gas sensor calibration. Journal of Sensors and Sensor Systems, 9(2): 411-424, 2020.

[3] T. Baur, J. Amann, C. Schultealbert und A. Schütze. Field Study of Metal Oxide Semiconductor Gas Sensors in Temperature Cycled Operation for Selective VOC Monitoring in Indoor Air. Atmosphere, 12(5), 2021.
[4] R. Benedix. Bauchemie, Einführung in die Chemie für Bauingenieure und Architekten: Chemie nichtmetallischanorganischer Baustoffe. Springer, Leipzig, 6 Auflage, 2015.

[5] B. de Lacy Costello, A. Amann, H. Al-Kateb, C. Flynn, W. Filipiak, T. Khalid, D. Osborne und N. Ratcliffe. A review of the volatiles from the healthy human body. Journal of Breath Research, 8(1), 2014.

[6] DIN. DIN ISO 16000-6 Innenraumluftverunreinigungen Teil 6: Bestimmung von VOC in der Innenraumluft und in Prüfkammern, Probenahme auf Tenax TA ${ }^{\circledR}$, thermische Desorption und Gaschromatographie mit MS oder MS-FID (ISO 16000-6:2011).

[7] N. Helwig, M. Schüler, C. Bur, A. Schütze und T. Sauerwald. Gas mixing apparatus for automated gas sensor characterization. Measurement Science and Technology, 25 (5), 2014.

[8] H. Hofmann und P. Plieninger. Bereitstellung einer Datenbank zum Vorkommen von flüchtigen organischen Verbindungen in der Raumluft). URL https: //www.umweltbundesamt.de/sites/default/files/medien/ publikation/long/3637.pdf(2008).

[9] H. Hofmann, G. Erdmann und A. Müller. Zielkonflikt energieeffiziente Bauweise und gute Raumluftqualität - Datenerhebung für flüchtige organische Verbindungen in der Innenraumluft von Wohn- und Bürogebäuden (Lösungswege). URL https://www.agoef.de/fileadmin/user_upload/dokumente/ forschung/AGOEF-Abschlussbericht_VOC_DB_IIbarrierefrei.pdf(2014).

[10] G. Korotcenkov und B. K. Cho. Instability of metal oxidebased conductometric gas sensors and approaches to stability improvement (short survey). Sensors and Actuators B: Chemical, 156(2):527-538, 2014.

[11] M. Leidinger, T. Sauerwald, W. Reimringer, G. Ventura und A. Schütze. Selective detection of hazardous VOCs for indoor air quality applications using a virtual gas sensor array. Journal of Sensors and Sensor Systems, 3(2):253-263, 2014.

[12] I. Paciência, J. Madureira, J. Rufo, A. Moreira und E. D. O. Fernandes. A systematic review of evidence and implications of spatial and seasonal variations of volatile organic compounds (VOC) in indoor human environments. Journal of Toxicology Environmental Health Part B, 19(2):47-64, 2016.

[13] P. N. Pegas, T. Nunes, C. A. Alves, J. R. Silva, S. L. A. Vieira, A. Caseiro und C. A. Pio. Indoor and outdoor characterisation of organic and inorganic compounds in city centre and suburban elementary schools of aveiro, portugal. Atmospheric Environment, 55:80-89, 2012.

[14] D. Rüffer, F. Hoehne und J. Bühler. New digital metal-oxide (mox) sensor platform. Sensors, 18(4), 2018.

[15] D. A. Sarigiannis. Combined or multiple exposure to health stressors in indoor built environments. WHO report for Europe, 2013.

[16] A. Schütze und T. Sauerwald. Dynamic operation of semiconductor sensors. In Semiconductor Gas Sensors. Woodhead Publishing, 2 Auflage, 2020.

[17] C. Schultealbert, J. Amann, T. Baur und A. Schütze. Measuring Hydrogen in Indoor Air with a Selective Metal Oxide Semiconductor Sensor. Atmosphere, 12(3), 2021. 